\title{
Enhanced Enzyme Activity after Incubation with Zinc Can Be Used to Distinguish Heterozygotes of Pompe's Disease
}

\author{
CHING-YUANG LIN \\ Departments of Medical Research and Pediatrics, Veterans General Hospital, Taipei, Taiwan, Republic of China
}

\begin{abstract}
In an attempt to identify heterozygotes for Pompe's disease, the acid $\alpha$-D-glucosidase activity at $\mathrm{pH} 4$ in phytohemagglutinin-stimulated lymphocytes was measured. In the standard assay an overlap in the enzyme activity between normal controls and obligate heterozygotes was demonstrated. When the assay was modified by adding zinc chloride into the incubation mixture, the enzyme activity of the lymphocyte homogenates did not change. However, the enzyme activity increased dramatically after intact lymphocytes were preincubated with zinc chloride. There was no more overlap in the acid $\alpha-\mathrm{D}$ glucosidase activity between normal controls and obligate heterozygotes when Iymphocytes were incubated $1 \mathrm{~h}$ with $10^{-4} \mathrm{~mol} / \mathrm{liter}$ zinc chloride or $2 \mathrm{~h}$ with $10^{-3}$ or $10^{-4} \mathrm{~mol} /$ liter zinc chloride. This modified enzyme activity should facilitate improved heterozygote detection. (Pediatr Res 23: 283-287, 1988)
\end{abstract}

\section{Abbreviations}

PHA, phytohemagglutinin

FCS, fetal calf serum

4-MU- $\alpha$-glc, 4-methylumbelliferyl- $\alpha$-D-glucopyranoside

Glycogen storage disease type II (Pompe's disease) (McKusick no. 23230) is an autosomal recessive disorder characterized by the lysosomal accumulation of glycogen $(1,2)$. The impaired glycogen degradation is due to a deficiency of acid $\alpha$-glucosidase (EC 3.2.1.20) (1-6). Several clinical forms of Pompe's disease that differ in age of onset, organ involvement, and progression of the disease have been recognized (1-7). In Taiwan Pompe's disease is the most common type of glycogen storage disease (79). Most cases have had the infantile form (generalized glycogenosis II) with symptoms appearing shortly after birth. Cardiomegaly, hepatomegaly, and muscular weakness are usually present. Patients typically die within the 1 st yr of life due to cardiac failure or respiratory tract complications. Therefore, the identification of both homozygous and heterozygous patients is important for genetic counseling and prenatal diagnosis. In our previous reports we established the normal reference range of acid $\alpha$-D-glucosidase levels in different age groups in this population which is useful not only for clinical cases but also for prenatal diagnosis (8). Often heterozygote detection is difficult. For heterozygotes identification studies measuring enzyme activ-

Received August 12, 1987; accepted November 4, 1987

Correspondence: Dr. Ching-Yuang Lin, Pediatric Research Laboratory, Department of Medical Research, Veterans General Hospital, Shih-Pai, Taipei, Taiwan, 11217 , R.O.C.

Supported by Grant NSC75-0412-B075-41 from the National Science Council of the Republic of China. ity from PHA-stimulated lymphocytes $(10,11)$, leukocytes $(12$, 13), cultivated fibroblasts (14), or skeletal muscles (15) have been reported. However, each method has its limitation. In an attempt to determine a more accurate method for the recognition of heterozygotes, we established an enzyme assay method using zinc stimulated intact lymphocytes which is superior to the method using PHA-stimulated lymphocytes.

\section{MATERIALS AND METHODS}

Subjects. From April 1981 to June 198618 families had one or more infant deaths with Pompe's disease (infantile generalized form). The criteria for diagnosis of Pompe's disease have been described previously (7-9). These criteria are: l) presence of clinical features of hypotonia, cardiomegaly, and hepatomegaly; 2) depressed ST segments, inverted $T$ waves, and a shortened P-R interval on electrocardiogram; 3) light microscopic and electron microscopic demonstration of lysosomal glycogen deposition; and 4) markedly deficient $\alpha$-glucosidase activity in lymphocytes, muscle cells, and/or cultured fibroblasts. Obligate heterozygotes from 14 of these families were studied; five families were consanguinous.

Lymphocyte separation. Lymphocytes were separated using a Ficoll-Hypaque density gradient as previously described $(7,8)$. The monocytes were removed with Petri dish adherence. The polymorphonuclear leukocytes were removed with $6 \%$ dextran.

PHA-stimulated lymphocytes. Lymphocytes were cultured in RPMI- 1640 containing $10 \%$ heat-inactivated FCS with $5 \mu \mathrm{g} / \mathrm{ml}$ PHA or without. Cultures were carried out for $72 \mathrm{~h}$ in a $5 \% \mathrm{CO}_{2}$ incubator at $37^{\circ} \mathrm{C}$. At the end of the culture lymphocytes were washed three times with isotonic saline and then the activity of $\alpha$-glucosidase was measured.

$\alpha$-D-glucosidase activity. The activity of acid $\alpha$-glucosidase was assayed with 4-MU- $\alpha$-glc (Koch-Light Laboratories, Colnbrook, England) as substrate by the method of Salafsky and Nadler (16) with the following modifications. Twenty- $\mu$ l cell suspensions (varying from $5 \times 10^{4}$ to $5 \times 10^{5}$ ) were incubated with $50 \mu \mathrm{l}$ of $1 \mathrm{mM}$ 4-MU- $\alpha$-glc in citrate phosphate buffer $\mathrm{pH} 4$ and 6 for 2 $h$ at $37^{\circ} \mathrm{C}$ with constant agitation. The reaction was terminated by adding $1 \mathrm{ml}$ of $400 \mathrm{mM}$ glycine- $\mathrm{NaOH}$ buffer, $\mathrm{pH} 10.5$. Fluorescence was determined in an Hitachi or an AmincoBowman spectrofluorometer with an excitation wavelength of $360 \mathrm{~nm}$ and an emission wavelength of $450 \mathrm{~nm}$. The standard contained $0.05 \mathrm{mM}(8.81 \mathrm{mg} /$ liter $)$ of 4-methylumbelliferone (Koch-Light) in $0.05 \mathrm{M}$ glycine carbonate buffer. Protein was determined by the method of Lowry et al. (17).

Acid $\alpha$-D-glucosidase activity from lymphocytes after incubation with zinc. To elucidate the effect of zinc, the acid $\alpha$-Dglucosidase activity in intact lymphocytes from four homozygotes, 28 heterozygotes, and 20 healthy controls were measured after being incubated with final concentration of $10^{-1}, 10^{-2}, 10^{-3}$, $10^{-4} \mathrm{~mol} /$ liter zinc chloride and incubated for 1,2 , and $16 \mathrm{~h}$. 
Acid $\alpha$-D-glucosidase activity from lymphocyte homogenates after incubation with zinc. For the study of the effect of zinc in $\mathrm{pH} 4$ citrate buffer on the $\alpha$-D-glucosidase in lymphocyte homogenates, homogenates from four homozygotes, 20 heterozygotes, and 20 healthy controls were incubated with final concentrations of $10^{-3}, 10^{-4}, 10^{-5}, 10^{-6}, 10^{-7}, 10^{-8}, 10^{-9} \mathrm{~mol} /$ liter zinc chloride with or without EDTA for 15 and $30 \mathrm{~min}$, then the acid $\alpha$-D-glucosidase activity was immediately determined.

Controls. The lymphocytes used as controls were separated with Ficoll-Hypaque density gradient from 20 age- and sexmatched healthy persons.

Statistical analysis. The differences between groups were assessed by the approximate $t$ test, or Student's $t$ test, depending on the equality of the variances of the two groups being compared.

\section{RESULTS}

Stimulated versus unstimulated. The lymphocyte acid $\alpha$-Dglucosidase activity increased after PHA stimulation in both heterozygotes or affected homozygotes but decreased in normal healthy controls (Fig. 1). Comparison of acid $\alpha$-D-glucosidase activity in PHA-stimulated lymphocytes both in normal healthy controls and heterozygotes showed no statistical difference.

Modified enzyme activity with the addition of zinc. The acid$\alpha$-D-glucosidase activity from lymphocyte homogenate after

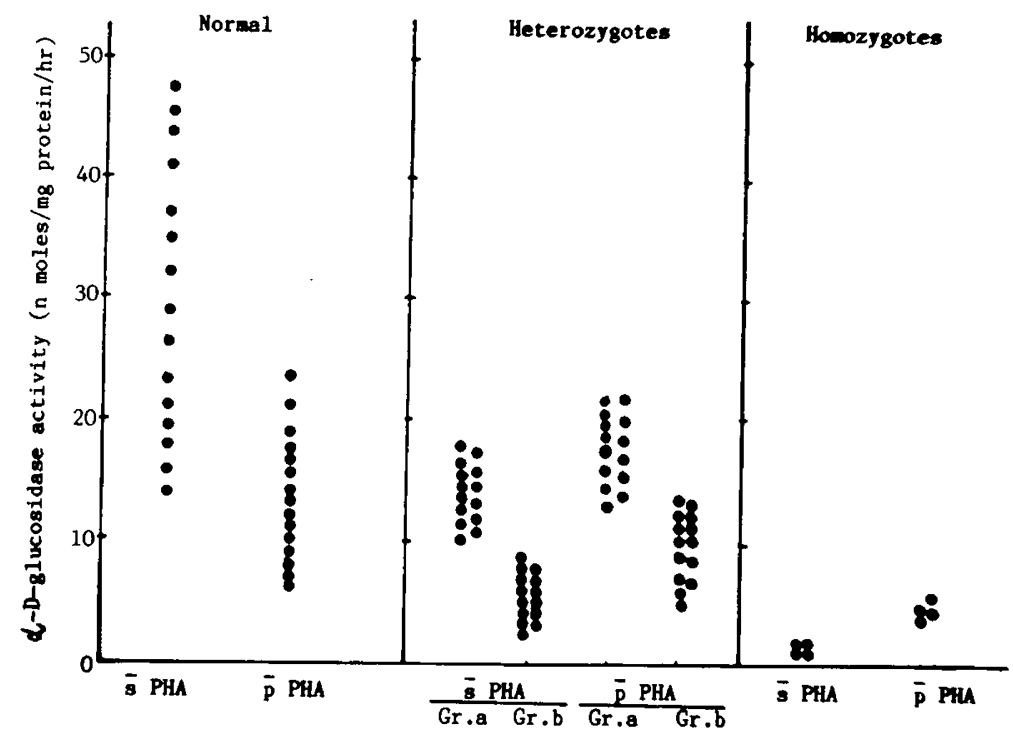

Fig. 1. Comparison of acid $\alpha$-D-glucosidase activity in PHA-stimulated lymphocytes among homozygous, heterozygous, and normal controls. The $\alpha$-D-glucosidase in heterozygotes can be divided into two groups, group a (Gr.a) (which is parent a in Tables 1 to 5) is slightly low; but group $\mathrm{b}(G r . b)$ (which is parent $\mathrm{b}$ in Tables 1 to 5 ) is very low. $\bar{s}$, without; $\bar{p}$, post.

Table 1. Activity of the acid $\alpha$-D-glucosidase* (pH 4) from lymphocyte homogenate after incubation with $\mathrm{ZnCl}_{2}$ for 15 min $[\mathrm{mean}$ $\pm S D$ (range)]

\begin{tabular}{|c|c|c|c|c|c|c|c|c|c|}
\hline \multirow{2}{*}{$\begin{array}{l}\text { Lymphocyte } \\
\text { homogenate }\end{array}$} & \multicolumn{7}{|c|}{ Homogenate incubation with $\mathrm{Zn}^{++}$(mol/liter) for $15 \mathrm{~min}$} & \multirow{2}{*}{$\begin{array}{c}\text { With } \\
\text { EDTA }\end{array}$} & \multirow[b]{2}{*}{ Nothing } \\
\hline & $10^{-3}$ & $10^{-4}$ & $10^{-5}$ & $10^{-6}$ & $10^{-7}$ & $10^{-8}$ & $10^{-9}$ & & \\
\hline $\begin{array}{l}\text { Normal } \\
(n=20)\end{array}$ & $\begin{array}{c}25.07 \\
\pm 4.62 \\
(20.52- \\
30.06)\end{array}$ & $\begin{array}{r}26.89 \\
\pm 5.48 \\
(20.46- \\
32.82)\end{array}$ & $\begin{array}{c}25.20 \\
\pm 6.24 \\
(18.82- \\
32.41)\end{array}$ & $\begin{array}{r}24.40 \\
\pm 7.66 \\
(26.48- \\
32.52)\end{array}$ & $\begin{array}{c}27.35 \\
\pm 5.56 \\
(21.42- \\
32.62)\end{array}$ & $\begin{array}{c}22.41 \\
\pm 5.42 \\
(16.74- \\
28.52)\end{array}$ & $\begin{array}{c}21.11 \\
\pm 6.12 \\
(14.84- \\
28.32)\end{array}$ & $\begin{array}{r}23.29 \\
\pm 10.96 \\
(12.88- \\
34.42)\end{array}$ & $\begin{array}{c}26.67 \\
\pm 5.34 \\
(18.42- \\
32.18)\end{array}$ \\
\hline
\end{tabular}

Heterozygotes $\uparrow$

\begin{tabular}{|c|c|c|c|c|c|c|c|c|c|}
\hline $\begin{array}{l}\text { Patient a } \\
(n=14)\end{array}$ & $\begin{array}{r}9.70 \\
\pm 2.34 \\
(6.42- \\
13.81)\end{array}$ & $\begin{array}{c}10.63 \\
\pm 3.61 \\
(6.32- \\
14.70)\end{array}$ & $\begin{array}{r}11.20 \\
\pm 3.84 \\
(6.82- \\
15.24)\end{array}$ & $\begin{array}{r}11.01 \\
\pm 4.21 \\
(6.38- \\
15.28)\end{array}$ & $\begin{array}{r}10.24 \\
\pm 3.76 \\
(6.42- \\
14.74)\end{array}$ & $\begin{array}{r}10.51 \\
\pm 4.04 \\
(6.34- \\
15.12)\end{array}$ & $\begin{array}{r}10.16 \\
\pm 3.68 \\
(6.14- \\
15.80)\end{array}$ & $\begin{array}{r}11.17 \\
\pm 1.06 \\
(9.72- \\
14.40)\end{array}$ & $\begin{array}{r}12.04 \\
\pm 2.06 \\
(10.42- \\
14.80)\end{array}$ \\
\hline $\begin{array}{l}\text { Patient b } \\
(n=14)\end{array}$ & $\begin{array}{r}3.92 \\
\pm 0.42 \\
(2.12- \\
4.86)\end{array}$ & $\begin{array}{r}4.11 \\
\pm 0.48 \\
(2.84- \\
4.78)\end{array}$ & $\begin{array}{r}4.68 \\
\pm 0.52 \\
(2.92- \\
5.02)\end{array}$ & $\begin{array}{r}4.11 \\
\pm 0.36 \\
(2.88 \\
4.74)\end{array}$ & $\begin{array}{r}4.07 \\
\pm 0.40 \\
(2.56- \\
4.82)\end{array}$ & $\begin{array}{r}3.69 \\
\pm 0.32 \\
(2.34- \\
4.41)\end{array}$ & $\begin{array}{r}3.67 \\
\pm 0.48 \\
(2.16- \\
4.32)\end{array}$ & $\begin{array}{r}3.42 \\
\pm 0.50 \\
(1.06- \\
5.43)\end{array}$ & $\begin{array}{r}3.48 \\
\pm 0.48 \\
(1.08 \\
5.62)\end{array}$ \\
\hline $\begin{array}{l}\text { Homozygotes } \\
\quad(n=4)\end{array}$ & $\begin{array}{c}0.20 \\
\pm 0.24 \\
(0.01- \\
0.80)\end{array}$ & $\begin{array}{c}0.21 \\
\pm 0.32 \\
(0.01- \\
0.90)\end{array}$ & $\begin{array}{r}0.24 \\
\pm 0.28 \\
(0.01- \\
0.84)\end{array}$ & $\begin{array}{r}0.22 \\
\pm 0.30 \\
(0.01- \\
0.88)\end{array}$ & $\begin{array}{r}0.18 \\
\pm 0.12 \\
(0.01- \\
0.72)\end{array}$ & $\begin{array}{r}0.18 \\
\pm 0.18 \\
(0.01- \\
0.74)\end{array}$ & $\begin{array}{r}0.17 \\
\pm 0.14 \\
(0.01- \\
0.70) \\
\end{array}$ & $\begin{array}{r}0.38 \\
\pm 0.12 \\
(0.10- \\
1.24)\end{array}$ & $\begin{array}{r}0.36 \\
\pm 0.14 \\
(0.10- \\
1.26)\end{array}$ \\
\hline
\end{tabular}

${ }^{*} \alpha$-D-Glucosidase was measured in $\mathrm{n} \mathrm{mol} / \mathrm{mg}$ protein $/ \mathrm{h}$.

$\dagger$ In our experience the $\alpha$-D-glucosidase in heterozygotes can be divided into two groups, one (parent a) is slightly low (>10 nmol/mg protein/h) and the other (parent $\mathrm{b})$ is very low $(<10 \mathrm{nmol} / \mathrm{mg}$ protein $/ \mathrm{h})$. 
Table 2. Activity of the acid $\alpha$-D-glucosidase* (pH 4) from lymphocyte homogenate after incubation with $\mathrm{ZnCl}$ for 30 min [mean \pm $S D$ (range)]

\begin{tabular}{|c|c|c|c|c|c|c|c|c|c|}
\hline \multirow{2}{*}{$\begin{array}{l}\text { Lymphocyte } \\
\text { homogenate }\end{array}$} & \multicolumn{7}{|c|}{ Homogenate incubation with $\mathrm{Zn}^{++}$(mol/liter) for $30 \mathrm{~min}$} & \multirow[b]{2}{*}{ With EDTA } & \multirow[b]{2}{*}{ Nothing } \\
\hline & $10^{-3}$ & $10^{-4}$ & $10^{-5}$ & $10^{-6}$ & $10^{-7}$ & $10^{-8}$ & $10^{-9}$ & & \\
\hline $\begin{array}{l}\text { Normal } \\
\qquad(n=20)\end{array}$ & $\begin{array}{c}23.51 \\
\pm 3.24 \\
(19.42- \\
27.34)\end{array}$ & $\begin{array}{c}25.97 \\
\pm 4.36 \\
(19.52- \\
30.84)\end{array}$ & $\begin{array}{c}26.59 \\
\pm 4.21 \\
(19.36- \\
31.32)\end{array}$ & $\begin{array}{c}28.68 \\
\pm 4.71 \\
(21.84- \\
33.72)\end{array}$ & $\begin{array}{c}31.78 \\
\pm 5.40 \\
(22.52- \\
37.56)\end{array}$ & $\begin{array}{r}26.31 \\
\pm 6.38 \\
(19.14- \\
34.36)\end{array}$ & $\begin{array}{c}24.81 \\
\pm 4.98 \\
(19.02- \\
30.16)\end{array}$ & $\begin{array}{r}23.29 \\
\pm 10.96 \\
(12.92- \\
36.82)\end{array}$ & $\begin{array}{c}26.67 \\
\pm 5.34 \\
(16.32- \\
35.80)\end{array}$ \\
\hline \multicolumn{10}{|l|}{ Heterozygotes $\dagger$} \\
\hline $\begin{array}{l}\text { Patient a } \\
(n=14)\end{array}$ & $\begin{array}{r}6.70 \\
+2.32 \\
(3.12- \\
9.18)\end{array}$ & $\begin{array}{r}7.17 \\
\pm 3.28 \\
(3.10- \\
10.82)\end{array}$ & $\begin{array}{r}7.56 \\
\pm 3.42 \\
(3.02- \\
11.54)\end{array}$ & $\begin{array}{r}8.68 \\
\pm 4.53 \\
(3.21- \\
14.32)\end{array}$ & $\begin{array}{r}9.83 \\
\pm 4.27 \\
(4.12- \\
15.38)\end{array}$ & $\begin{array}{r}8.60 \\
\pm 3.83 \\
(3.42- \\
12.82)\end{array}$ & $\begin{array}{r}8.88 \\
\pm 2.87 \\
(3.04- \\
11.52)\end{array}$ & $\begin{array}{c}11.17 \\
\pm 1.06 \\
(10.14- \\
14.21)\end{array}$ & $\begin{array}{c}12.05 \\
\pm 2.06 \\
(10.02- \\
15.32)\end{array}$ \\
\hline $\begin{array}{l}\text { Patient b } \\
(n=14)\end{array}$ & $\begin{array}{r}2.06 \\
\pm 0.14 \\
(1.04- \\
3.14)\end{array}$ & $\begin{array}{r}2.24 \\
\pm 0.30 \\
(1.08- \\
3.16)\end{array}$ & $\begin{array}{r}2.38 \\
\pm 0.26 \\
(1.06- \\
3.04)\end{array}$ & $\begin{array}{r}2.82 \\
\pm 0.30 \\
(1.04- \\
3.86)\end{array}$ & $\begin{array}{r}3.38 \\
\pm 0.14 \\
(1.12- \\
5.14)\end{array}$ & $\begin{array}{r}2.62 \\
\pm 0.32 \\
(1.10- \\
4.08)\end{array}$ & $\begin{array}{c}2.22 \\
\pm 0.18 \\
(1.11- \\
3.44)\end{array}$ & $\begin{array}{c}4.49 \\
\pm 1.48 \\
(1.01- \\
5.46)\end{array}$ & $\begin{array}{r}4.83 \\
\pm 1.28 \\
(1.06- \\
6.02)\end{array}$ \\
\hline $\begin{array}{l}\text { Homozygotes } \\
\quad(n=4)\end{array}$ & $\begin{array}{r}0.06 \\
\pm 0.02 \\
(0.01- \\
0.52) \\
\end{array}$ & $\begin{array}{r}0.08 \\
\pm 0.04 \\
(0.01- \\
0.64)\end{array}$ & $\begin{array}{r}0.11 \\
\pm 0.08 \\
(0.01- \\
0.72) \\
\end{array}$ & $\begin{array}{r}0.16 \\
\pm 0.14 \\
(0.01- \\
0.82) \\
\end{array}$ & $\begin{array}{r}0.17 \\
\pm 0.20 \\
(0.01- \\
0.90) \\
\end{array}$ & $\begin{array}{r}0.10 \\
\pm 0.21 \\
(0.01- \\
0.91) \\
\end{array}$ & $\begin{array}{c}0.08 \\
\pm 0.14 \\
(0.01- \\
0.80) \\
\end{array}$ & $\begin{array}{r}0.22 \\
\pm 0.10 \\
(0.01- \\
1.02) \\
\end{array}$ & $\begin{array}{r}0.32 \\
\pm 0.12 \\
(0.01- \\
1.22) \\
\end{array}$ \\
\hline
\end{tabular}

* $\alpha$-D-glucosidase was measured in $\mathrm{nmol} / \mathrm{mg}$ protein $/ \mathrm{h}$.

$\dagger$ In our experience the $\alpha$-D-glucosidase in heterozygotes can be divided into two groups, one (patient a) is slightly low $(>10 \mathrm{nmol} / \mathrm{mg} \mathrm{protein} / \mathrm{h}$ ) and the other (patient $\mathrm{b})$ is very low $(<10 \mathrm{nmol} / \mathrm{mg}$ protein $/ \mathrm{h})$.

Table 3. Activity of the acid $\alpha$-D-glucosidase* (pH 4) from lymphocytes after incubation with $\mathrm{ZnCl} 2$ for $1 \mathrm{~h}[\mathrm{mean} \pm \mathrm{SD}(\mathrm{range})]$

\begin{tabular}{|c|c|c|c|c|c|c|}
\hline \multirow[b]{2}{*}{ Lymphocytes } & \multicolumn{4}{|c|}{ Whole cells incubation with $\mathrm{Zn}^{++}$(mol/liter) for $1 \mathrm{~h}$} & \multirow{2}{*}{$\begin{array}{c}\text { Without } \\
\mathrm{Zn}^{++}\end{array}$} & \multirow{2}{*}{$\begin{array}{l}\text { With } \\
\text { EDTA }\end{array}$} \\
\hline & $10^{-1}$ & $10^{-2}$ & $10^{-3}$ & $10^{-4}$ & & \\
\hline $\begin{array}{l}\text { Normal } \\
(n=20)\end{array}$ & $\begin{array}{l}22.45+, a \\
\pm 8.26 \\
(18.28- \\
32.52)\end{array}$ & $\begin{array}{c}26.63^{b} \\
\pm 8.82 \\
(14.24- \\
36.72)\end{array}$ & $\begin{array}{c}37.98^{c f} \\
\pm 8.74 \\
(27.52- \\
48.62)\end{array}$ & $\begin{array}{c}46.83^{d, g} \\
\pm 10.95 \\
(24.12- \\
58.42)\end{array}$ & $\begin{array}{c}29.09^{e} \\
\pm 16.82 \\
(12.36- \\
50.31)\end{array}$ & $\begin{array}{c}27.84^{h} \\
\pm 15.68 \\
(13.52- \\
48.86)\end{array}$ \\
\hline \multicolumn{7}{|l|}{ Heterozygotes $\$$} \\
\hline $\begin{array}{l}\text { Patient a } \\
(n=14)\end{array}$ & $\begin{array}{r}14.17 \\
\pm 7.61 \\
(6.36- \\
24.72)\end{array}$ & $\begin{array}{c}19.76 \\
\pm 6.12 \\
(11.42- \\
27.56)\end{array}$ & $\begin{array}{c}24.04^{f} \\
\pm 6.71 \\
(14.62- \\
34.82)\end{array}$ & $\begin{array}{c}17.61^{g} \\
\pm 8.44 \\
(9.42- \\
23.64)\end{array}$ & $\begin{array}{r}17.74 \\
\pm 4.65 \\
(10.26- \\
22.62)\end{array}$ & $\begin{array}{c}16.82 \\
\pm 5.27 \\
(10.12- \\
23.54)\end{array}$ \\
\hline $\begin{array}{l}\text { Patient b } \\
(n=14)\end{array}$ & $\begin{array}{c}3.99^{a} \\
\pm 4.13 \\
(0.08- \\
9.14)\end{array}$ & $\begin{array}{c}5.48^{b} \\
+5.39 \\
(0.08- \\
12.42)\end{array}$ & $\begin{array}{r}10.57^{c} \\
\pm 10.34 \\
(0.09- \\
21.52)\end{array}$ & $\begin{array}{c}5.58^{d} \\
\pm 4.80 \\
(1.12- \\
12.14)\end{array}$ & $\begin{array}{c}3.41^{e} \\
\pm 2.24 \\
(1.02- \\
7.42)\end{array}$ & $\begin{array}{r}3.04^{h} \\
\pm 2.82 \\
1.06- \\
8.42)\end{array}$ \\
\hline $\begin{array}{l}\text { Homozygotes } \\
\qquad(n=4)\end{array}$ & $\begin{array}{c}0.34 \\
\pm 0.12 \\
(0.01- \\
0.90)\end{array}$ & $\begin{array}{c}0.36 \\
\pm 0.14 \\
(0.01- \\
0.92)\end{array}$ & $\begin{array}{r}0.38 \\
\pm 0.16 \\
(0.01- \\
0.94)\end{array}$ & $\begin{array}{c}0.32 \\
\pm 0.10 \\
0.01- \\
0.94)\end{array}$ & $\begin{array}{c}0.38 \\
\pm 0.12 \\
0.01- \\
1.06) \\
\end{array}$ & $\begin{array}{r}0.31 \\
\pm 0.16 \\
0.01- \\
1.08) \\
\end{array}$ \\
\hline
\end{tabular}

* $\alpha$-D-Glucosidase was measured in $\mathrm{nmol} / \mathrm{mg}$ protein $/ \mathrm{h}$.

$\dagger$ Mean values in the same column followed by a common letter are significantly different $(p<0.001)$ by Student's or approximate $t$ test.

\$In our experience the $\alpha$-D-glucosidase in heterozygotes can be divided into two groups, one (parent a) is slightly low ( $>10 \mathrm{nmol} / \mathrm{mg} \mathrm{protein} / \mathrm{h}$ ) and the other (parent $\mathrm{b})$ is very low $(<10 \mathrm{nmol} / \mathrm{mg}$ protein $/ \mathrm{h})$. 
being incubated with different concentrations of zinc chloride for 15 and $30 \mathrm{~min}$ is shown in Tables 1 and 2. Comparison of acid $\alpha$-D-glucosidase activity among each group incubated with and without zinc chloride revealed no statistical difference.

The acid $\alpha$-D-glucosidase activity from whole intact lymphocytes increased after incubation with zinc chloride for 1 (Table 3) and 2 (Fig. 2; Table 4) $\mathrm{h}$ but not after $16 \mathrm{~h}$. When the incubation time was set at $1 \mathrm{~h}$, the maximal increased enzyme activity was at the zinc chloride concentration of $10^{-4} \mathrm{~mol} / \mathrm{liter}$. With zinc preincubation the group of heterozygotes with enzyme activity above and below $10 \mathrm{nmol} / \mathrm{mg}$ protein/h from the normal

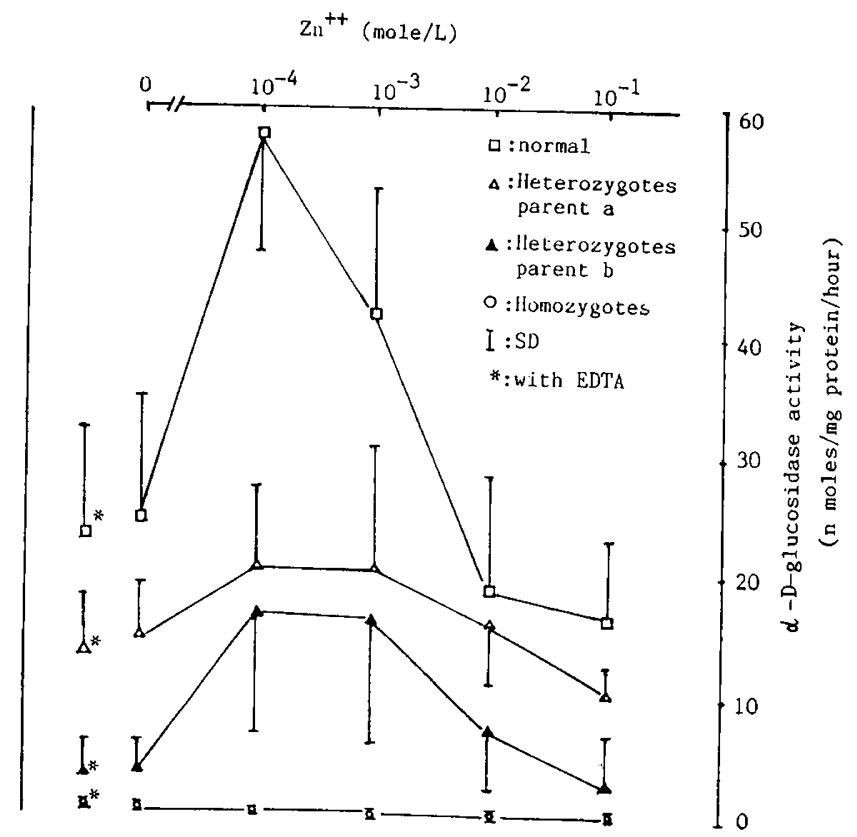

Fig. 2. The activity of the acid $\alpha$-D-glucosidase (pH 4) from lymphocytes after incubation with $\mathrm{ZnCl}_{2}$ for $2 \mathrm{~h}$. controls could be distinguished clearly (Table 3 ). When the incubation time increased to $2 \mathrm{~h}$, the maximal enzyme activity was still at the zinc chloride concentration of $10^{-4} \mathrm{~mol} / \mathrm{liter}$. There was also a statistical difference between heterozygotes with enzyme activity above $10 \mathrm{nmol} / \mathrm{mg}$ protein/h group and normal controls at $10^{-3}$ and $10^{-4} \mathrm{~mol} /$ liter zinc chloride (Table 4). After the incubation time increased to $16 \mathrm{~h}$, the zinc chloride was unable to distinguish between heterozygotes and normal controls (Table 5).

\section{DISCUSSION}

Pompe's disease, type II glycogenosis, is the most common type of glycogenosis in Taiwan (7-9). The disease involves mainly Southern Chinese and is rare in Northern Chinese (8) and Japanese (18). In our estimation the frequency of heterozygotes in Taiwan is about 0.5 to $1 \%$ which is similar to the Netherlands and Belgium (3). This is a high frequency hereditary disease. Therefore, genetic counseling and heterozygotes detection are very important. Hirschhorn et al. (10) and Taniguchi et al. (11) have reported that the assay of acid $\alpha$-D-glucosidase activity in PHA-stimulated lymphocytes is useful in the detection of heterozygotes of this disease. However, we found that there was overlap in the acid $\alpha$-D-glucosidase activity of PHA-stimulated lymphocytes between normal controls and obligate heterozygotes. Therefore, we tried to improve the sensitivity of the method for a better differentiation of the heterozygotes using metallic ions including $\mathrm{Ag}^{+}, \mathrm{Hg}^{++}, \mathrm{Zn}^{++}, \mathrm{Cu}^{++}$, and $\mathrm{Cd}^{++}$. No remarkable improvement in the enzyme activity of lymphocyte homogenate was found by $\mathrm{Zn}^{++}, \mathrm{Cu}^{++}, \mathrm{Cd}^{++}$, and EDTA. Instead, a strong inhibition by $\mathrm{Ag}^{+}$and $\mathrm{Hg}^{++}$was noted. It is surprising that $\alpha$-D-glucosidase activity from intact lymphocytes increased markedly after incubation with zinc. Furthermore, the acid $\alpha$-D-glucosidase activity of lymphocytes incubated $1 \mathrm{~h}$ with $10^{-4} \mathrm{~mol} /$ liter zinc chloride or $2 \mathrm{~h}$ with $10^{-3}$ or $10^{-4} \mathrm{~mol} / \mathrm{liter}$ zinc chloride did not overlap between the normal controls and obligate heterozygotes. This result suggests that the zinc-enhanced enzyme activity may be useful for heterozygote detection.

Table 4. Activity of the acid $\alpha$-D-glucosidase* ( $p H$ 4) from lymphocytes after incubation with $Z n C l_{2}$ for $2 h$ [mean $\pm S D$ (range)]

\begin{tabular}{|c|c|c|c|c|c|c|}
\hline \multirow[b]{2}{*}{ Lymphocytes } & \multicolumn{4}{|c|}{ Whole cells incubation with $\mathrm{Zn}^{++}$(mol/liter) for $2 \mathrm{~h}$} & \multirow{2}{*}{$\begin{array}{c}\text { Without } \\
\mathrm{Zn}^{++}\end{array}$} & \multirow{2}{*}{$\begin{array}{c}\text { With } \\
\text { EDTA }\end{array}$} \\
\hline & $10^{-1}$ & $10^{-2}$ & $10^{-3}$ & $10^{-4}$ & & \\
\hline $\begin{array}{l}\text { Normal } \\
(n=20)\end{array}$ & $\begin{array}{l}16.43 \dagger^{+}, a \\
\pm 6.68 \\
(6.52- \\
28.82)\end{array}$ & $\begin{array}{c}18.81^{b} \\
\pm 9.52 \\
(8.12- \\
30.56)\end{array}$ & $\begin{array}{c}41.96^{c, f} \\
\pm 11.32 \\
(28.24- \\
58.92)\end{array}$ & $\begin{array}{c}57.54^{d, g} \\
\pm 10.28 \\
(42.42- \\
70.18)\end{array}$ & $\begin{array}{c}24.65^{e} \\
\pm 10.82 \\
(12.42- \\
36.92)\end{array}$ & $\begin{array}{c}23.42^{h} \\
\pm 9.63 \\
(12.14- \\
36.56)\end{array}$ \\
\hline \multicolumn{7}{|l|}{ Heterozygotes $\$$} \\
\hline $\begin{array}{l}\text { Parent a } \\
(n=14)\end{array}$ & $\begin{array}{c}10.36 \\
\pm 2.27 \\
(7.24- \\
14.32)\end{array}$ & $\begin{array}{c}16.14 \\
\pm 5.07 \\
(10.41- \\
22.72)\end{array}$ & $\begin{array}{c}20.59^{f} \\
\pm 9.84 \\
10.82- \\
31.26)\end{array}$ & $\begin{array}{c}21.06^{g} \\
\pm 6.59 \\
(12.92- \\
28.32)\end{array}$ & $\begin{array}{r}14.74 \\
\pm 4.65 \\
(9.82- \\
20.48)\end{array}$ & $\begin{array}{c}13.67 \\
\pm 5.32 \\
(8.12- \\
20.72)\end{array}$ \\
\hline $\begin{array}{l}\text { Parent b } \\
(n=\quad)\end{array}$ & $\begin{array}{c}2.85^{a} \\
\pm 4.18 \\
(0.92- \\
0.48)\end{array}$ & $\begin{array}{c}7.25^{b} \\
\pm 5.11 \\
(0.92- \\
14.54)\end{array}$ & $\begin{array}{c}16.61^{c} \\
\pm 10.42 \\
(4.82- \\
30.12)\end{array}$ & $\begin{array}{c}16.96^{d} \\
\pm 9.88 \\
(6.52- \\
28.42)\end{array}$ & $\begin{array}{c}3.41^{e} \\
\pm 2.55 \\
(0.92- \\
7.42)\end{array}$ & $\begin{array}{c}3.08^{h} \\
\pm 2.47 \\
(0.94- \\
7.62)\end{array}$ \\
\hline $\begin{array}{l}\text { Homozygotes } \\
\quad(n=4)\end{array}$ & $\begin{array}{r}0.22 \\
\pm 0.10 \\
(0.01- \\
0.90) \\
\end{array}$ & $\begin{array}{r}0.26 \\
\pm 0.14 \\
(0.01- \\
0.92) \\
\end{array}$ & $\begin{array}{c}0.30 \\
\pm 0.10 \\
(0.01- \\
0.92) \\
\end{array}$ & $\begin{array}{c}0.30 \\
\pm 0.20 \\
(0.01- \\
0.94) \\
\end{array}$ & $\begin{array}{r}0.36 \\
\pm 0.10 \\
(0.01- \\
1.02) \\
\end{array}$ & $\begin{array}{r}0.29 \\
\pm 0.09 \\
(0.01- \\
1.00)\end{array}$ \\
\hline
\end{tabular}

${ }^{*} \alpha$-D-Glucosidase was measured in $\mathrm{nmol} / \mathrm{mg}$ protein $/ \mathrm{h}$.

$\dagger$ Mean values in the same column followed by a common letter are significantly different $(p<0.001)$ by Student's or approximate $t$ test.

$\ddagger$ In our experience the $\alpha$-D-glucosidase in heterozygotes can be divided into two groups, one (parent a) is slightly low $(>10 \mathrm{nmol} / \mathrm{mg} \mathrm{protein} / \mathrm{h}$ ) and the other (parent $\mathrm{b}$ ) is very low $(<10 \mathrm{nmol} / \mathrm{mg}$ protein $/ \mathrm{h})$. 
Table 5. Activity of the acid $\alpha$-D-glucosidase* (pH 4) from lymphocytes after incubation with $\mathrm{ZnCl}$ for $16 \mathrm{~h}[\mathrm{mean} \pm \mathrm{SD}$ (range)]

\begin{tabular}{|c|c|c|c|c|c|c|}
\hline \multirow[b]{2}{*}{ Lymphocytes } & \multicolumn{4}{|c|}{ Whole cells incubation with $\mathrm{Zn}^{++}(\mathrm{mol} /$ liter) for $16 \mathrm{~h}$} & \multirow{2}{*}{$\begin{array}{c}\text { Without } \\
\mathrm{Zn}^{++}\end{array}$} & \multirow{2}{*}{$\begin{array}{l}\text { With } \\
\text { EDTA }\end{array}$} \\
\hline & $10^{-1}$ & $10^{-2}$ & $10^{-3}$ & $10^{-4}$ & & \\
\hline $\begin{array}{l}\text { Normal } \\
(n=20)\end{array}$ & $\begin{array}{r}2.06 \\
\pm 3.36 \\
(0.10- \\
5.82)\end{array}$ & $\begin{array}{c}6.31 \uparrow^{+a, b} \\
\pm 4.19 \\
(1.02- \\
11.72)\end{array}$ & $\begin{array}{c}26.14^{c, d} \\
\pm 11.05 \\
(12.41- \\
48.52)\end{array}$ & $\begin{array}{c}27.50^{\gamma .8} \\
\pm 14.86 \\
12.62- \\
45.82)\end{array}$ & $\begin{array}{c}19.65^{h} \\
\pm 8.82 \\
(10.42- \\
30.52)\end{array}$ & $\begin{array}{c}18.42^{i} \\
\pm 7.46 \\
(10.64- \\
28.42)\end{array}$ \\
\hline \multicolumn{7}{|l|}{ Heterozygotes $\ddagger$} \\
\hline $\begin{array}{l}\text { Parent a } \\
(n=14)\end{array}$ & $\begin{array}{r}1.57 \\
\pm 1.92 \\
(0.14- \\
3.12)\end{array}$ & $\begin{array}{r}5.93 \\
\pm 6.07 \\
(0.94- \\
12.73)\end{array}$ & $\begin{array}{c}11.45^{e} \\
\pm 5.17 \\
(5.72- \\
19.42)\end{array}$ & $\begin{array}{r}9.93 \\
\pm 6.13 \\
(2.14- \\
17.92)\end{array}$ & $\begin{array}{c}13.41 \\
\pm 4.25 \\
(8.42- \\
19.48)\end{array}$ & $\begin{array}{r}12.82 \\
\pm 4.94 \\
(7.24 \\
18.22)\end{array}$ \\
\hline $\begin{array}{l}\text { Parent b } \\
(n=14)\end{array}$ & $\begin{array}{c}0.39 \\
\pm 0.11 \\
(0.21- \\
1.04)\end{array}$ & $\begin{array}{c}2.47^{b} \\
+1.74 \\
(0.90- \\
5.12)\end{array}$ & $\begin{array}{c}6.35^{d} \\
\pm 2.61 \\
(1.01- \\
10.52)\end{array}$ & $\begin{array}{c}6.55^{g} \\
\pm 5.18 \\
(0.94- \\
12.81)\end{array}$ & $\begin{array}{r}4.74 \\
\pm 3.65 \\
(0.92- \\
9.02)\end{array}$ & $\begin{array}{r}4.14 \\
\pm 3.21 \\
(0.96 \\
9.41)\end{array}$ \\
\hline $\begin{array}{l}\text { Homozygotes } \\
\quad(n=4)\end{array}$ & $\begin{array}{r}0.12 \\
\pm 0.08 \\
(0.01- \\
0.90)\end{array}$ & $\begin{array}{c}0.16^{a} \\
\pm 0.10 \\
(0.01- \\
0.91)\end{array}$ & $\begin{array}{c}0.26^{c, e} \\
\pm 0.12 \\
(0.01- \\
0.94)\end{array}$ & $\begin{array}{c}0.26^{f} \\
\pm 0.10 \\
(0.01- \\
1.02)\end{array}$ & $\begin{array}{c}0.28^{h} \\
\pm 0.06 \\
(0.01- \\
1.06) \\
\end{array}$ & $\begin{array}{c}0.20^{i} \\
\pm 0.08 \\
(0.01- \\
1.08)\end{array}$ \\
\hline
\end{tabular}

* $\alpha$-D-Glucosidase was measured in $\mathrm{nmol} / \mathrm{mg}$ protein $/ \mathrm{h}$.

$\dagger$ Mean values in the same column followed by a common letter are significantly different $(p<0.001)$ by Student's or approximate $t$ test.

$\ddagger$ In our experience the $\alpha$-D-glucosidase in heterozygotes can be divided into two groups, one (parent a) is slightly low ( $>10 \mathrm{nmol} / \mathrm{mg} \mathrm{protein} / \mathrm{h}$ ) and the other (parent $\mathrm{b})$ is very low $(<10 \mathrm{nmol} / \mathrm{mg}$ protein $/ \mathrm{h})$.

\section{REFERENCES}

1. McKusick VA 1983 Mendelian Inheritance in Man, 6th ed. Johns Hopkins University Press, Baltimore

2. Pompe JC 1932 Over idiopatische hypertrophie van het hart. Ned Jijdschr Geneskd 76:304-311

3. Loonen MCB 1979 The Variability of Pompe's Disease, 1st ed. Grafische Verzorging, Davids Decor Alblasserdam, p 98

4. Reuser AJJ, Koster JF, Hoogeveen A, Galjaar H 1978 Biochemical, immunological, and cell genetic studies in glycogenosis type II. Am J Hum Genet 30:132-143.

5. Angelini C, Engel AG 1972 Comparative study of acid maltase deficiency. Arch Neurol 26:344-349

6. Loon MCB, Schram AW, Korter JF, Niermeijer MF, Busch HFM, Martin JJ, Brouwer-Kelder B, Mekes W, Slee RG, Tager JM 1981 Identification of heterozygotes for glycogenosis 2 (acid maltase deficiency). Clin Genet 19:5563

7. Lin CY, Hwang B, Hsiao KJ, Jin YR 1986 Study of $\alpha$-D-glucosidase activity in patients with Pompe's disease. J Formosan Med Assoc 85:766-770

8. Lin CY, Hwang B, Hsiao KJ, Jin YR 1987 Pompe's disease in Chinese and the prenatal diagnosis by determination of $\alpha$-glucosidase activity. $J$ Interited Metab Dis 10:11-17

9. Hwang B. Meng CCL, Lin CY, Hsu HC 1986 Clinical analysis of five infants with glycogen storage disease of the heart-Pompe's disease. Jap Heart $J$
$27: 25-34$.

10. Hirschhorn K, Nadler HL, Waithe WI, Brown BI, Hirschhorn R 1969 Pompe's disease: detection of heterozygotes by lymphocyte stimulation. Science 166:1632-1633

11. Taniguchi N, Kato E, Yoshida H, Iwaki S, Ohki T, Koizumi S 1978 Alphaglucosidase activity in human leukocytes: Choice of lymphocytes for the diagnosis of Pompe's disease and the carrier state. Clin Chim Acta 89:293299

12. Williams HE 1966 Alpha-glucosidase activity in human leukocytes. Biochim Biophys Acta 124:34-38

13. Koster JF, Slee RG, Hülsmann WC 1974 The use of leukocytes as an acid in the diagnosis of a variant of glycogen storage disease type II (Pompe's disease). Clin Chem Acta 51:319-325

14. Nitowsky HM, Grunfeld A 1967 Lysosomal alpha-glucosidase in type II glycogenosis: activity in leukocytes and cell cultures in relation to genotype. J Lab Clin Med 69:472-484

15. Engel AG, Gomez MR 1970 Acid maltase levels in muscle in heterozygous acid maltase deficiency and in non-weak neuromuscular disease controls. $J$ Neurol Neurosurg Psychiat 33:801-804

16. Salafsky IS, Nadler HL 1971 Alpha-1,4-glucosidase activity in Pompe's disease. J Pediatr 79:794-798

17. Lowry OH, Rosebrough NJ, Farr AL, Randall RJ 1951 Protein measurement with the Folin phenol reagent. J Biol Chem 193:265-270

18. Nakamura L 1983 Glycogenoses. Jpn J Pediatr 15 (suppl):764-767, 1983 